www.nature.com/pj

\title{
Hierarchical assembly of CdS nanoparticles in polymer particles with phase separation structures
}

\author{
Hiroshi Yabu ${ }^{1,2}$, Ayako Endo ${ }^{1}, K$ azutaka Koike ${ }^{1}, K_{i w a m u}$ Motoyoshi ${ }^{1}$, Takeshi Higuchi ${ }^{3}$ and \\ Masatsugu Shimomura ${ }^{1,3}$
}

The preparation of size-controlled CdS nanoparticles in block copolymer micelles and formation of composite particles of CdS nanoparticles and polymers are described in this paper. Uniformly sized CdS nanoparticles with different emission wavelengths were successfully synthesized. In addition, composite Janus particles, unidirectionally stacked lamellae particles and onion particles were formed by combining CdS nanoparticles and polymer blend or block copolymer systems. This approach provides a simple route for the preparation of functional organic/inorganic composite particles.

Polymer Journal (2011) 43, 301-305; doi:10.1038/pj.2010.137; published online 19 January 2011

Keywords: block copolymer; CdS nanoparticles; micelles; polymer blends; self-assembly

\section{INTRODUCTION}

Inorganic polymer composite nanoparticles have attracted considerable interest because of their potential applications in photonics, electronics and biotechnology. ${ }^{1,2}$ By using the high conductivity, reflectivity and mechanical stability of inorganic materials, polymer particles have been functionalized by nanoscale hybridization with inorganic materials. Among the various inorganic nanomaterials, semiconductor nanoparticles are attractive because of their unique absorption and fluorescence properties, which are based on their quantum size effect. ${ }^{3}$ These quantum dots (QDs) show strong fluorescence, and their absorption and fluorescence profiles can be controlled by changing their sizes. ${ }^{4-7}$ These properties allow for a wide variety of practical applications, including imaging probes, ${ }^{8}$ sensors ${ }^{9}$ and solar cells. ${ }^{10}$ Recently, precise control of QD arrangements has attracted considerable interest because periodically arranged QDs can be used as a QD laser, ${ }^{11}$ which functions with a pumping light of very low threshold power.

Several processes have been demonstrated for the introduction of QDs into photonic crystals, which is one of the most promising ways to realize QD lasers. Some studies have shown that QDs can be electrochemically deposited within the voids of assembled colloidal crystals. ${ }^{12-15}$ Alternatively, QDs have been incorporated into submicron spheres using layer-by-layer assembly. ${ }^{16,17}$ In these examples, the coupling between QD emission and photonic crystal band gaps has been demonstrated. Recently, Moffitt and co-workers showed that multistep self-assembly of QDs and block copolymers enables the preparation of submicron-sized particles containing QDs and the creation of hierarchically structured photonic crystals. ${ }^{18}$ This process has advantages on the processability and applicability of various materials. However, the QD-QD interparticle distances and the three-dimensional arrangement of QDs in individual submicron particles cannot yet be precisely controlled, and these parameters will strongly affect the optical properties of photonic materials.

We have reported that polymer particles with phase-separation structures can be prepared by evaporating a good solvent from a solution of block copolymers or polymer blends that also contain a poor solvent (self-organized precipitation method). ${ }^{19}$ Polymer particles with unique nanostructures have been prepared by this simple evaporation method, including one-dimensionally stacked lamellar, onion, Janus and core-shell structures. ${ }^{20-25}$ Recently, it was reported that the inner structure of polymer blend particles could be controlled by the hydrophobicity of blended polymers. ${ }^{26}$ We have also found that block copolymer-stabilized Au nanoparticles can be introduced into polymer blend particles by the self-organized precipitation method. ${ }^{27,28}$

In this report, we describe the control of a special arrangement of CdS nanoparticles in submicron-sized polymer particles by using the self-assembly of polymer blends and block copolymers. The CdS nanoparticles were successfully synthesized in amphiphilic block copolymer micelle templates, and CdS nanoparticles and polymer composite nanoparticles with various phase separation structures were prepared by the self-organized precipitation method. The control of the inner structure of the CdS QD-polymer composite particles is discussed. 


\section{EXPERIMENTAL PROCEDURE}

The polymers used in this experiment are listed in Table 1. All polymers were purchased from Polymer Source, Inc (Quebec, Canada). Cadmium acetate monohydrate and sodium sulfide were purchased from Aldrich, St Louis, MO, USA.

CdS nanoparticles can be simply synthesized in block copolymer micelles. Koh et al. ${ }^{29}$ have reported that poly(styrene-block-2-vinylpyridine) (PS-b$\mathrm{P} 2 \mathrm{VP}$ ) forms micelles in a toluene solution; thus, CdS nanoparticles were synthesized by the simple addition of $\mathrm{Cd}$ ions and a sulfur source (Scheme 1i). PS-b-P2VP was dissolved in anhydrous toluene to prepare a $5.0-\mathrm{mg} \mathrm{ml}^{-1}$ solution in a three-necked flask equipped with a shrenk tube and septa. Cadmium acetate monohydrate was added to the toluene solution of PS-b$\mathrm{P} 2 \mathrm{VP}$ micelles, and the solution was then stirred for $2 \mathrm{~h}$ to load the $\mathrm{Cd}$ ions into PS-b-P2VP micelles. Sodium sulfide was dissolved in Millipore membranefiltered water (MilliQ, Millipore, Billerica, MA, USA) to prepare a saturated aqueous solution. The aqueous solution of sodium sulfide was added to the toluene solution of Cd-ion-loaded PS-b-P2VP micelles. The molar ratio of cadmium acetate to sodium sulfide is shown in Table 2. After $24 \mathrm{~h}$ of stirring, the solution was centrifuged ( 3000 r.p.m., $30 \mathrm{~min}$ ) to remove water and large precipitates, and the toluene phase was corrected.

Toluene was evaporated from the nanoparticle dispersion, and a $0.1 \mathrm{mg} \mathrm{ml}^{-1}$ tetrahydrofuran (THF) dispersion of the nanoparticles was prepared. Polystyrene (PS), polyisoprene (PI) and poly(styrene-block-isoprene) (PS-b-PI)

Table 1 Polymers used in this experiment

\begin{tabular}{lccc}
\hline Polymers & $M n_{(A)}\left(\mathrm{kg} \mathrm{mol}^{-1}\right)$ & $M n_{(B)}\left(\mathrm{kg} \mathrm{mol}^{-1}\right)$ & $M w / M n$ \\
\hline PS-b-P2VP & 44 & 8.4 & 1.12 \\
PS-b-PI-1 & 141.5 & 80.6 & 1.07 \\
PS-b-PI-2 & 318 & 512 & 1.15 \\
PS & 131 & - & 1.05 \\
PI & - & 18.5 & 1.03 \\
\hline
\end{tabular}

Abbreviations: PI, polyisoprene; PS, polystyrene; PS-b-PI, poly(styrene-block-isoprene); PS-bP2VP, poly(styrene-block-2-vinylpyridine).

$\mathrm{Mn}(\mathrm{A})$ and $\mathrm{Mn}(\mathrm{B})$ are the number average molecular weights of poly(A-block-B) in the case of the block-copolymers. were dissolved in THF at a concentration of $0.1 \mathrm{mg} \mathrm{m}^{-1}$. The THF dispersion of nanoparticles and the THF solution of polymers were mixed, and $1 \mathrm{ml}$ of water was slowly added to $1 \mathrm{ml}$ of the mixed solution in a glass test tube (Scheme 1ii). THF was evaporated from the sample solution in a test tube at $25^{\circ} \mathrm{C}$ placed in a water bath for 2 days. After complete evaporation of the THF, $400 \mu \mathrm{l}$ of a $0.2 \%$ aqueous solution of $\mathrm{OsO}_{4}$ was added to the same amount of the resultant aqueous dispersion of composite particles in an Eppendorf tube to stain and crosslink the PI moieties. After $2 \mathrm{~h}$ of incubation, the stained particles were purified by three cycles of ultracentrifugation (12000 r.p.m., $5^{\circ} \mathrm{C}, 15 \mathrm{~min}$ ), the supernatant removed and the particles washed with MilliQ water. The synthesized nanoparticles and composite particles were observed using a transmission electron microscope (H-7650, Hitachi, Hitachinaka, Japan). For transmission electron microscope observation, a sample was prepared by casting a $1-\mu \mathrm{l}$ dispersion of nanoparticles onto a $\mathrm{Cu}$ grid with a ultraviolet- $\mathrm{O}_{3}$-treated elastic carbon membrane. Micelles were observed using a field emission scanning electron microscope (S-5200, Hitachi) after depositing the micelles onto a $\mathrm{Si}$ substrate and sputtering Os onto the sample. Elemental analysis of the nanoparticles was performed using an X-ray photoelectron spectrometer (JPS-9200, JEOL, Tokyo, Japan; Al Ka X-rays with an energy of $1486.6 \mathrm{eV}$ were used with a pass energy of $10 \mathrm{eV}$.). After casting the dispersion of nanoparticles on a silicon substrate, the sample was calcined at $400{ }^{\circ} \mathrm{C}$ for $3 \mathrm{~h}$ to remove organic compounds. The optical properties of the

\section{Table 2 Conditions for the CdS nanoparticle preparation and} nanoparticle sizes

\begin{tabular}{lcccc}
\hline Entry & $\mathrm{Cd}(\mathrm{Oac})_{2}$ unit $^{\mathrm{a}}$ & $\mathrm{Na}_{2} \mathrm{~S}$ unit & Size $(\mathrm{nm})^{\mathrm{b}}$ & Spectra in Figure 2 \\
\hline 1 & 1 & 1 & 3.5 & $\mathrm{a}, \mathrm{d}$ \\
2 & 0.5 & 1 & 2.0 & $\mathrm{~b}$ \\
3 & 0.1 & 1 & 1.5 & $\mathrm{c}$ \\
4 & 1 & 5 & 3.3 & $\mathrm{e}$ \\
5 & 1 & 10 & 2.7 & $\mathrm{f}$
\end{tabular}

a Molar ratio against total poly(2-vinylpyridine) monomer unit of PS-b-P2VP.

bMeasured from transmission electron microscope images of CdS nanoparticles.

(i) preparation of CdS NP@PS-b-P2VP micelle

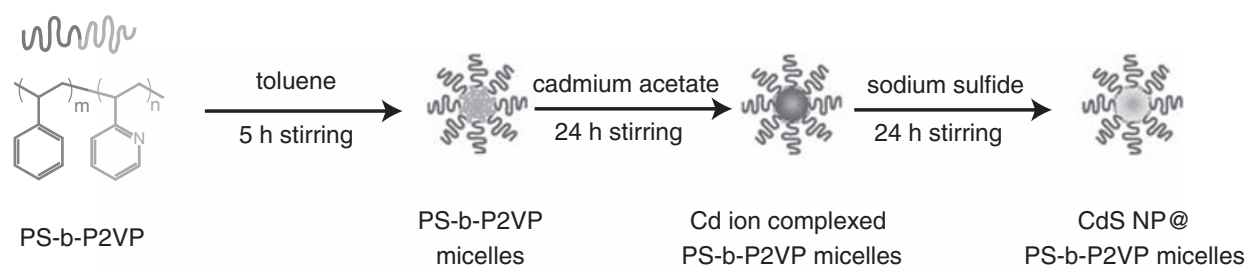

(ii) preparation of polymers/CdS NP@PS-b-P2VP micelle composite particles

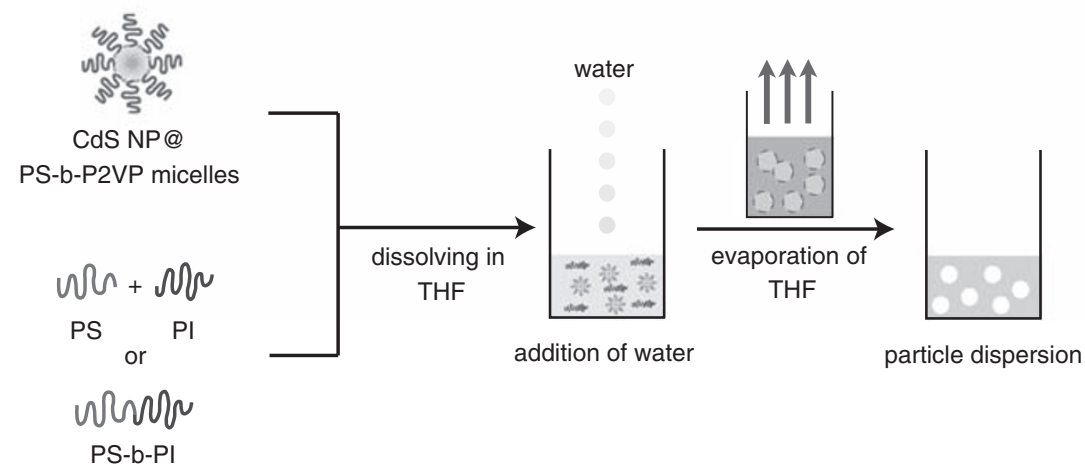

Scheme 1 Schematic illustration of the synthesis of CdS nanoparticles in block copolymer micelles (i) and preparation of composite particles (ii). PI, polyisoprene; PS, polystyrene; PS-b-P2VP, poly(styrene-block-2-vinylpyridine); THF, tetrahydrofuran. 
nanoparticles were measured using an ultraviolet-vis spectrometer and a fluorescence spectrometer.

\section{RESULTS AND DISCUSSION}

Figure 1A shows a typical transmission electron microscope image of CdS nanoparticles prepared in PS-b-P2VP micelles. A densely packed and uniformly sized CdS nanoparticle array was observed. The size of the PS-b-P2VP micelle is determined by the spacing between the CdS nanoparticles in the transmission electron microscope image; thus, the average diameter of the PS-b-P2VP micelles is $40 \mathrm{~nm}$. The size of micelles is also checked by field emission scanning electron microscope (see Supplementary Information). The average size of the CdS nanoparticles was $3.5 \mathrm{~nm}$, and the size distribution was $<20 \%$ in all cases. Figure 1B shows the X-ray photoelectron spectra of CdS nanoparticles prepared in PS-b-P2VP micelles for both wide and narrow scans. In the wide-scan spectrum, clear peaks attributed to the $\mathrm{C} 1 \mathrm{~s}, \mathrm{~N}$ 1s and $\mathrm{O} 1 \mathrm{~s}$ of PS-b-P2VP and the native oxide of the Si substrate are observed at 300,420 and $520 \mathrm{eV}$, respectively. In the narrow scan spectrum, peaks attributed to the $\mathrm{Cd} 3 \mathrm{~d} 3 / 2$ and $3 \mathrm{~d} 5 / 2$ of the CdS nanoparticles are observed at 416 and $409 \mathrm{eV}$, respectively. These results indicate that $\mathrm{Cd}$ is successfully incorporated into PS-bP2VP micelles.

It has been reported that $\mathrm{CdS}$ nanoparticles emit in the visible wavelength range when they are excited by ultraviolet light. As shown in Figure $2 \mathrm{Aa}$, strong emission around $\lambda=600 \mathrm{~nm}$ was observed from the toluene solution of the $\sim 3.5$-nm-diameter CdS QDs described in the previous paragraph. The size of CdS nanoparticles can be controlled by changing the amount of Cd source (Table 2) that is added during synthesis. The nanoparticle size decreased from 3.5 to $2.5 \mathrm{~nm}$ with a decrease in the cadmium acetate concentration, and the emission peaks of the CdS nanoparticles also blue-shifted from yellow to whitish blue with decreasing particle size (Figures $2 \mathrm{Ab}$ and $\mathrm{Ac}$, and inset photographs). Figure $2 \mathrm{~B}$ shows spectra of the $\mathrm{CdS}$ nanoparticles prepared by changing the concentration of sodium sulfide. The same spectral and size changes of the nanoparticles were observed when the concentration of sodium sulfide was changed. The particle diameters changed from 2.7 to $3.5 \mathrm{~nm}$ with the increase in sodium sulfide concentration.

There are three main factors governing the emission spectra of CdS nanoparticles: the quantum size effect, interband recombination and defect bands. ${ }^{30}$ It is well known that emission spectra of nanosized semiconductor particles blue-shift as the size decreases because of the quantum size effect. These experimental results suggest that the quantum size effect of CdS nanoparticles makes the emission peak shift. The smaller particles (Figures 2Ac and Bf) show multiple emission peaks, which are attributed to the three different band types of CdS nanoparticles: a higher energy interband recombination
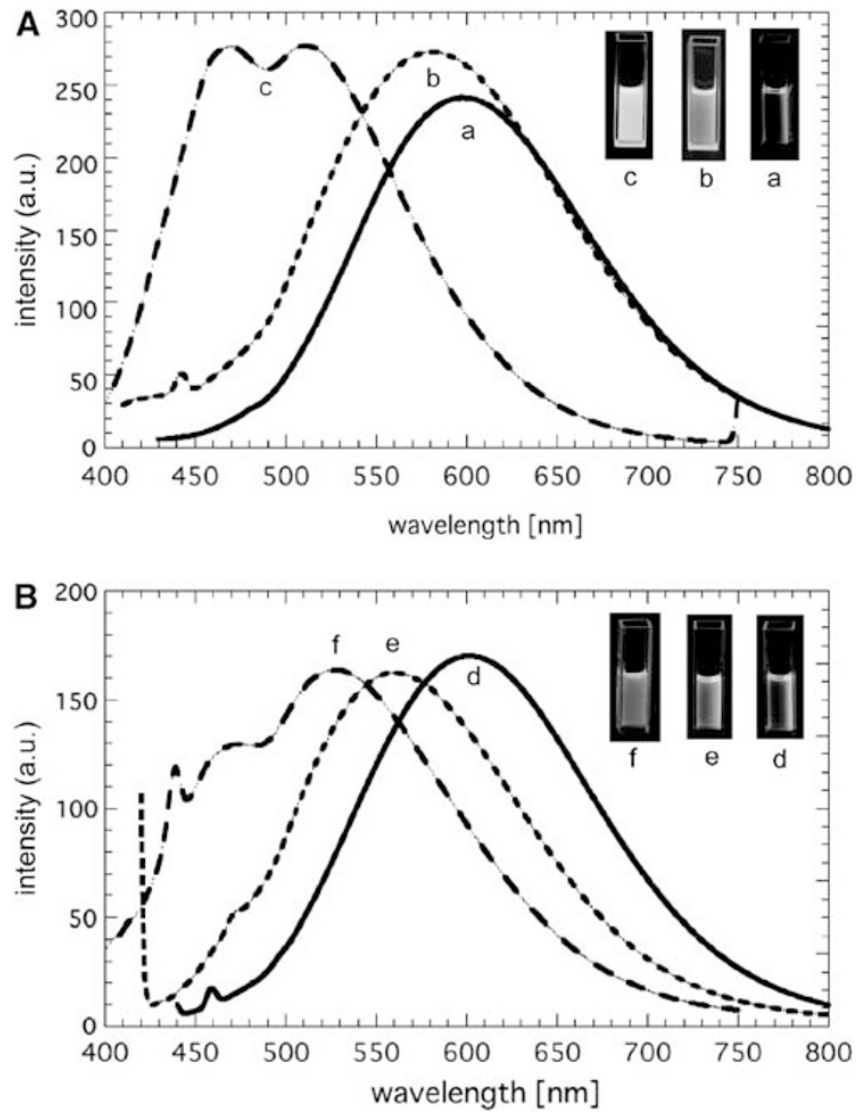

Figure 2 Emission spectra from CdS nanoparticles prepared by changing the $\mathrm{Cd}$ acetate concentration (A) and sodium sulfide concentration (B) (Ex. at $\lambda=350 \mathrm{~nm}$ ). The inset images are photographs of fluoresence from a toluene solution of CdS nanoparticles.
A

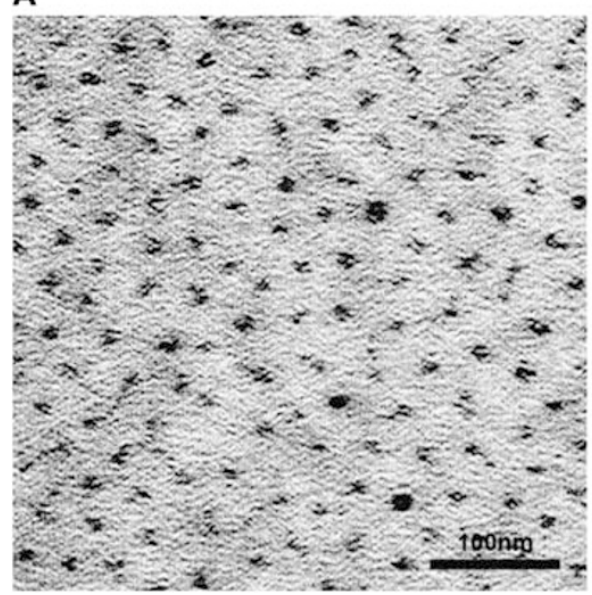

B

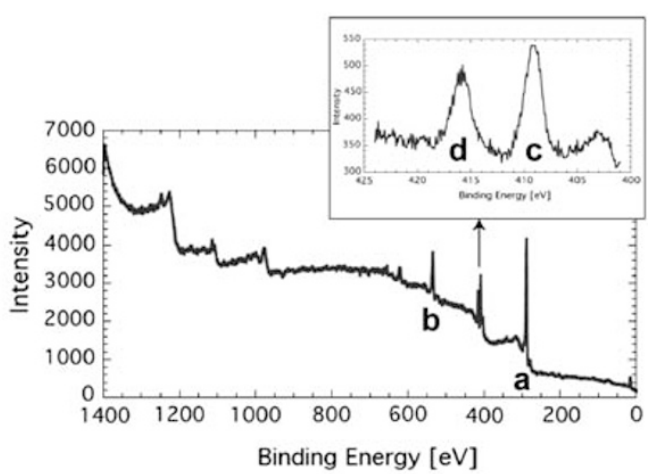

Figure 1 A typical transmission electron microscope image of CdS nanoparticles (A) and X-ray photoelectron spectrometer spectra of the CdS nanoparticles (B). 
and two types of lower energy defect bands. ${ }^{31}$ Figure 2Ac shows only the emission from defect bands, and Figure 2Bf shows emission from the above-mentioned three bands. These results also support the formation of CdS nanoparticles in PS-b-P2VP micelles.

Figure 3 shows three examples of composite particles containing CdS nanoparticles. Figure 3a shows composite particles of PS-b-P2VPstabilized CdS nanoparticles, PS and PI. The darker region of the particle indicates $\mathrm{PI}$ moieties stained with $\mathrm{OsO}_{4}$, and the lighter region

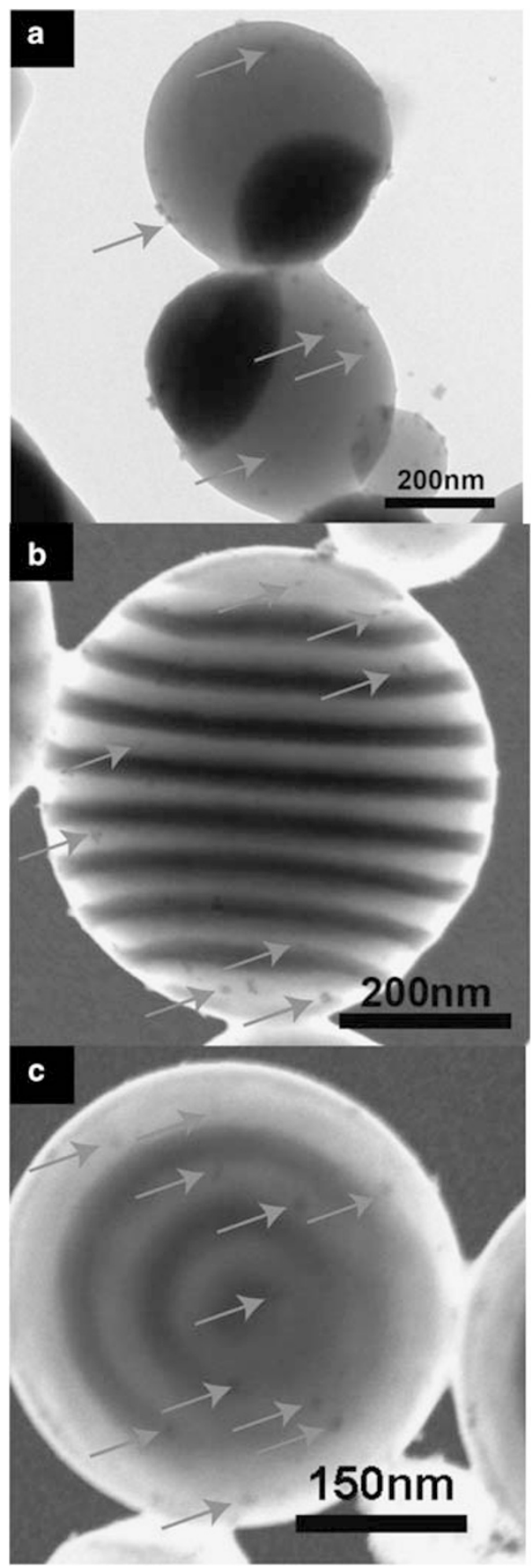

Figure $3 \mathrm{~A}$ transmission electron microscope image of composite particles of polystyrene (PS), polyisoprene (PI) and CdS nanoparticles (a) and a dark-field scanning transmission electron microscope (STEM) images of composite particles of PS-b-PI and CdS nanoparticles (b), (c). Red arrows indicate the CdS nanoparticles being introduced into polymer particles. is attributed to PS moieties. A clear Janus structure is observed. As shown in a previous report, ${ }^{26}$ PS and PI have similar solubility parameters; thus, these two polymers simultaneously precipitate as blend particles into the poor solvent. After precipitation, a Janus-type phase separation structure spontaneously forms to minimize the interface between the two phases. In this experiment, black dots, which are CdS nanoparticles, were observed only in the light PS region (red arrows). Because CdS nanoparticles were encapsulated in PS-bP2VP micelles, the CdS nanoparticles were selectively introduced into the PS phase.

Two types of composite particles were observed in the PS-b-PI block copolymer and PS-b-P2VP-stabilized CdS nanoparticles: one is a unidirectionally stacked lamellae structure and the other is an onionlike structure (Figures $3 \mathrm{~b}$ and c). Because PS-b-PI-1 and PS-b-PI-2 are symmetric diblock copolymers, they form a lamellar structure in their bulk states. ${ }^{32}$ The unidirectionally stacked lamellae and onion-like structures are types of 'isomers' based on the lamellar structure of the nanoparticles. As we reported previously, ${ }^{20-25,33}$ these two structures can be controlled by changing the solution concentration and by thermal annealing. Moreover, the periodicity of the phase separation can be controlled by changing the molecular weights of block copolymers within particles. In this experiment, the average periodicities between the PS-PS phases of PS-b-PI-1 and PS-b-PI-2 are 45 and $60 \mathrm{~nm}$, respectively. In these two cases, CdS nanoparticles are selectively introduced only into the PS phase (red arrows). As a result, the interparticle distances and arrangement of CdS nanoparticles are controlled by the periodicities and morphologies of the phase separation structures formed in the particles. From these results, it is clear that hierarchic composite particles of CdS nanoparticles and polymers were successfully prepared.

\section{CONCLUSION}

We show the preparation of size-controlled CdS nanoparticles in block copolymer micelles and the formation of composite particles of CdS nanoparticles and polymers. The emission spectra of the CdS nanoparticles were controlled by changing the particle sizes. Composite Janus, unidirectionally stacked lamellae and onion particles were formed by a combination of CdS nanoparticles and polymer blend or CdS nanoparticles and block copolymer systems.

These results indicate that novel functional composite particles with hierarchically assembled structures can be prepared by a simple mixing and evaporation process. These particles can be used in a wide variety of practical applications in the fields of photonics and electronics.

\section{ACKNOWLEDGEMENTS}

We thank Dr Y Matsuo, Hokkaido Innovation through NanoTechnology Support (HINTS), Hokkaido University, for helping with the X-ray photoelectron spectrometer measurement.

1 Caruso, F. (ed.) Colloids and Colloid Assemblies (Wiley-VCH, Weinheim, 2004).

2 Mao, Z., Xu, H. \& Wang, D. Molecular mimetic self-assembly of colloidal particles. Adv. Func. Mater. 20, 1053 (2010).

3 Yang, P. \& Murase, N. Preparation-condition dependence of hybrid SiO2-coated CdTe nanocrystals with intense and tunable photoluminescence. Adv. Func. Mater. 20, 1258 (2010).

4 Banerjee, R., Jayakrishnan, R. \& Ayyub, P. Effect of the size-induced structural transformation on the band gap in CdS nanoparticles. J. Phys. Condens. Matter. 12, 10647 (2000).

5 Chen, S., Truax, L. A. \& Sommers, J. M. Alkanethiolate-protected PbS nanoclusters: synthesis, spectroscopic and electrochemical studies. Chem. Mater. 12, 3864 (2000). 
6 Yu, W. W., Qu, L., Guo, W. \& Peng, X. Experimental determination of the extinction coefficient of CdTe, CdSe, and CdS nanocrystals. Chem. Mater. 15, 2854 (2003).

7 Jing, L., Yang, C., Quao, R., Niu, M., Du, M., Wang, D. \& Gao, M. Highly fluorescent CdTe@SiO2 particles prepared via reverse microemulsion method. Chem. Mater. 22, 420 (2010).

8 Hu, S.- H., Kuo, K.- T., Tung, W.- L., Liu, D.- M. \& Chen, S.- Y. A multifunctional nanodevice capable of imaging, magnetically controlling, and in situ monitoring drug release. Adv. Func. Mater. 19, 3396 (2009).

9 Zamir, E., Lommerse, P. H. M., Kinkhabwala, A., Grecco, H. E. \& Bastiaens, P. I. H. Fluorescence fluctuations of quantum-dot sensors capture intracellular protein interaction dynamics. Nat. Methods 7, 295 (2010).

10 Debnath, R., Tang, J., Barkhouse, D. A., Wang, X., Pattantyus-Abraham, A. G., Brzozowski, L., Levina, L. \& Sargent, E. H. Ambient-processed colloidal quantum dot solar cells via individual pre-encapsulation of nanoparticles. J. Am. Chem. Soc. 132, 5952 (2010)

11 Noumura, M., Kumagai, N., Iwamoto, S., Ota, Y. \& Arakawa, Y. Laser oscillation in a strongly coupled single-quantum-dot-nanocavity system. Nat. Phys. 6, 279 (2010).

12 Blanco, A., Lopez, C., Mayoral, R., Miguez, H., Meseguer, F., Mifsud, A. \& Herrero, J. CdS photoluminescence inhibition by a photonic structure. Appl. Phys. Lett. 73, 1781 (1998).

13 Vlasov, Y. A., Yao, N. \& Norris, D. J. Synthesis of photonic crystals for optical wavelengths from semiconductor quantum dots. Adv. Mater. 11, 165 (1999).

14 Yu, A., Meiser, F., Cassagneau, T. \& Caruso, F. Fabrication of polymer-nanoparticle composite inverse opals by a one-step electrochemical co-deposition process. Nano. Lett. 4, 177 (2004).

15 Lodahl, P., van Driel, A. F., Nikolaev, I. S., Irman, A., Overgaag, K., Vanmaekelbergh, D. \& Vos, W. L. Controlling the dynamics of spontaneous emission from quantum dots by photonic crystals. Nature 430, 654 (2000).

16 Rogach, A. L., Susha, A., Caruso, F., Sukhorukov, G., Kornowski, A., Kershaw, S. Möhwald, H., Eychmueller, A. A. \& Weller, H. 3-D colloidal photonic crystals prepared from sub- $\mu \mathrm{m}$-sized polystyrene latex spheres pre-coated with luminescent polyelectrolyte/nanocrystal shells. Adv. Mater. 12, 333 (2000)

17 Wang, D., Rogach, A. L. \& Caruso, F. Composite photonic crystals from semiconductor nanocrystal/polyelectrolyte-coated colloidal spheres. Chem. Mater. 15, 2724 (2003).

18 Yusuf, H., Kim, W.- G., Lee, D. H., Aloshyna, M., Brolo, A. G. \& Moffitt, M. G. A hierarchical self-assembly route to three-dimensional polymer-quantum dot photonic arrays. Langmuir 23, 5251 (2007).

19 Yabu, H., Higuchi, T., Ijiro, K. \& Shimomura, M. Spontaneous formation of polymer nanoparticles by good-solvent evaporation as non-equilibrium process. Chaos 14 047505 (2005).
20 Yabu, H., Higuchi, T. \& Shimomura, M. Unique phase separation structures on blockcopolymer nanoparticles. Adv. Mater. 17, 2062 (2005).

21 Higuchi, T., Yabu, H. \& Shimomura, M. Preparation of hollow nanoparticles of amphiphilic block-copolymers. J. Nanosci. Nanotech. 7, 856 (2007).

22 Higuchi, T., Yabu, H. \& Shimomura, M. Irreversible lamella-disorder phase transition in block-copolymer particles. Koubunshi Ronbunshu 64, 177, 856 (2007).

23 Higuchi, T., Yabu, H., Onoue, S., Kunitake, T. \& Shimomura, M. Preparation of lamellastructured block-copolymer particles and their irreversible lamella-disorder phase taransition. Colloids Surf. A 313-314, 87 (2008).

24 Higuchi, T., Motoyoshi, K., Tajima, A., Yabu, H. \& Shimomura, M. Frustrated phases of block copolymers in their nanoparticles. Angew. Chem. Int. Ed. 48, 5125 (2009).

25 Yabu, H., Motoyoshi, K., Higuchi, T. \& Shimomura, M. Hierarchical structures in AB/AC type diblock-copolymer blend particles. Phys. Chem. Chem. Phys. 12, 11944 (2010).

26 Motoyoshi, K., Higuchi, T., Yabu, H. \& Shimomura, M. Static and dynamic control of phase separation structures in nanoparticles of polymer blends. Soft Matter 6, 1253 (2010).

27 Yabu, H., Koike, K., Motoyoshi, K., Higuchi, T. \& Shimomura, M. Preparation of coreshell organic-inorganic nanocomposite particles based on phase separation of polymer blend. Chem. Lett. 38, 964 (2009).

28 Yabu, H., Koike, K., Motoyoshi, K., Higuchi, T. \& Shimomura, M. A novel route for fabricating metal-polymer composite particles with phase separation structures. Macromol. Rap. Commun. 31, 1267 (2010).

29 Koh, H.- D., Kang, N.- G. \& Lee, J.- S. Location control of Au/CdS nanoparticles in block copolymer micelles. Langmuir 23, 11425 (2007).

30 Cheyne, R. B. \& Moffitt, M. G. Controllable organization of quantum dots into mesoscale wires and cables via interfacial block copolymer self-assembly. Macromolecules 40, 2046 (2007).

31 Rodríguez-Fragoso, P., González de la Cruz, G., Tomás, S. A., Mendoza-Alvarez, J. G. \& Zelaya Angel, O. Photoluminescence of CdS nanoparticles embedded in a starch matrix. J. Lumines. 130, 1128 (2010).

32 Higuchi, T., Tajima, A., Yabu, H. \& Shimomura, M. Spontaneous formation of polymer nanoparticles with inner phase separation structures. Soft Matter 4, 1302 (2008).

33 Higuchi, T., Motoyoshi, K., Sugimori, H., Jinnai, H., Yabu, H. \& Shimomura, M. Phase transition and phase transformation in block copolymer nanoparticles. Macromol. Rap. Commun. 31, 1773 (2010).

Supplementary Information accompanies the paper on Polymer Journal website (http://www.nature.com/pj) 